\title{
Evidence for the Impact of Diet, Fluid Intake, Caffeine, Alcohol and Tobacco on Lower Urinary Tract Symptoms: A Systematic Review
}

\author{
Catherine S. Bradley, MD, MSCE ${ }^{1}$, Bradley A. Erickson, MD, MS, FACS ${ }^{1}$, Emily E. \\ Messersmith, $\mathrm{PhD}^{2}$, Anne P. Cameron, $\mathrm{MD}^{3}$, H. Henry Lai, MD ${ }^{4}$, Karl J. Kreder, MD, MBA ${ }^{1}$, \\ Claire C. Yang, MD ${ }^{5}$, Robert M. Merion, MD, FACS ${ }^{2}$, Tamara G. Bavendam, MD, MS $^{6}$, and \\ Ziya Kirkali, MD ${ }^{6}$ for the Symptoms of Lower Urinary Tract Dysfunction Research Network \\ (LURN) \\ ${ }^{1}$ University of lowa Carver College of Medicine, lowa City, IA \\ ${ }^{2}$ Arbor Research Collaborative for Health, Ann Arbor, MI \\ ${ }^{3}$ University of Michigan, Ann Arbor, MI \\ ${ }^{4}$ Washington University School of Medicine, St. Louis, MO \\ ${ }^{5}$ University of Washington, Seattle WA \\ ${ }^{6}$ National Institute of Diabetes and Digestive and Kidney Diseases, National Institutes of Health, \\ Bethesda, MD
}

\begin{abstract}
Purpose-Foods, fluid intake, caffeine, alcohol, and tobacco may influence lower urinary tract symptoms (LUTS). Changes in these potentially modifiable non-urologic factors (NUF) are often suggested to improve LUTS. To better understand the relationship of NUFs with LUTS, we performed a systematic literature review to examine, grade, and summarize reported associations between LUTS and diet, fluid intake, caffeine, tobacco and alcohol use.
\end{abstract}

Materials and Methods-We performed PubMed searches for eligible articles providing evidence on associations between one or more NUF and LUTS. A modified Oxford system was used to grade the evidence.

\begin{abstract}
Results-We reviewed 110 articles covering diet $(n=28)$, fluid intake $(n=21)$, caffeine $(n=20)$, alcohol $(\mathrm{n}=26)$ and tobacco use $(\mathrm{n}=44)$. The evidence grade was generally low (6\% level $1,24 \%$ level 2,11\% level 3; 59\% level 4). Fluid intake was associated with urinary frequency and urgency in men and women. Modest alcohol use was associated with less likelihood of benign prostatic hyperplasia (BPH) diagnosis and fewer LUTS in men. LUTS associations with food, caffeine, and tobacco were inconsistent.
\end{abstract}

Conclusions-Evidence for associations between LUTS and diet, fluid intake, caffeine, alcohol and tobacco use is sparse and mostly observational. However, there is evidence of associations

Corresponding Author Catherine S. Bradley, MD, MSCE, Professor of ObGyn, Urology and Epidemiology, Department of Obstetrics and Gynecology, University of Iowa Hospitals \& Clinics, 200 Hawkins Drive, Iowa City, IA 52242, catherine-bradley@uiowa.edu. 
between increased fluid intake and urinary frequency/urgency, and between modest alcohol intake and decreased BPH diagnosis and LUTS. Given the importance of these NUF to daily life, and their perceived impact on LUTS, higher quality evidence is needed.

\section{Keywords}

Lower urinary tract symptoms; systematic review

\section{Introduction}

Lower urinary tract symptoms (LUTS) are common and bothersome, affecting 20-50\% of men and women and negatively impacting health-related quality of life (QOL) ${ }^{1-3}$. Patients seeking care for LUTS are frequently instructed to modify daily behaviors to reduce symptoms. For example, providers may recommend patients change fluid intake, or use less caffeine or alcohol ${ }^{4}$. The quantity and quality of evidence to support such recommendations is unclear. While typically low risk, lifestyle changes may be obtrusive to patients' lives and increase anxiety or stress. What patients eat, drink, and ingest depends on culture, region, employment, socioeconomic status, and other factors. These behaviors are part of the daily human experience, and as such, a better understanding of their impact on LUTS is critical.

The Symptoms of Lower Urinary Tract Dysfunction Research Network (LURN) is a National Institute of Diabetes and Digestive and Kidney Diseases (NIDDK)-supported cooperative network with objectives to improve the measurement of LUTS and identify important LUTS subtypes ${ }^{5}$. In conceptualizing the scope of lower urinary tract dysfunction and its resultant symptoms, we considered multiple potential explanatory factors that may contribute to LUTS, including non-urologic factors (NUF), such as diet, fluid intake and caffeine, alcohol and tobacco use.

The objectives of this study were to identify, grade, and summarize peer-reviewed literature examining associations between diet, fluid intake, caffeine, alcohol and tobacco use and LUTS. In addition to identifying evidence-based associations between these factors and LUTS, results will help identify gaps where future efforts may be focused.

\section{Methods}

This systematic review was designed to answer the question: "Are diet, fluid intake, and caffeine, alcohol and tobacco use associated with the prevalence and/or severity of LUTS in men and women?". The review used findings from randomized clinical trial (RCT), cohort, case control, case series, and cross-sectional studies that could provide evidence related to these associations. Research focused on bladder pain and conditions such as interstitial cystitis/bladder pain syndrome were excluded. This systematic review was based on the Preferred Reporting Items for Systematic Reviews and Meta-Analyses (PRISMA) guidelines ${ }^{6}$.

PubMed searches were developed with assistance from a health science librarian. Five separate searches were performed to identify publications studying associations between LUTS and each of the five NUF. A search "string" was developed for LUTS and for each 
factor including MESH terms and key words for text searches and limited to English language publications (Supplemental Table 1).

All citations and abstracts were screened using previously developed eligibility criteria (Table 1). When the initial screener was unsure whether a citation should be included, a second investigator reviewed it. If uncertainty persisted, the citation was included for additional review at the full text stage. Each article considered eligible after screening was reviewed (full text) by two investigators. All articles confirmed eligible were assigned a level of evidence by both reviewers, using a system based on the Oxford Centre for Evidencebased Medicine Level of Evidence scale (2009 version) and International Consultation on Urological Diseases, modified to include cross-sectional studies as level $4 \mathrm{~b}$ evidence (Table $2)^{7,8}$. If initial grades differed, investigators arrived at a grade by consensus.

Data from each article were reviewed and abstracted using a standard form. Information collated included study design, population, LUTS outcome (e.g. overactive bladder [OAB]), NUF exposure (e.g. caffeine), the summary measure of association and type of analysis performed. Meta-analyses were not performed given the heterogeneous study designs, outcomes and exposures identified.

\section{Results}

Electronic searches were performed through January 4, 2016. Results of the searches, screening and selection process, and reasons for exclusion are presented in Tables 1 and 3. We reviewed 110 unique articles in the areas of diet $(n=28)$, fluid intake $(n=21)$, caffeine $(n=20)$, alcohol $(n=26)$ and tobacco $(n=44)$. Twenty-two articles contributed results on more than one factor. The evidence grade was generally low (6\% level 1, 24\% level 2, $11 \%$ level 3 , and 59\% level 4). A summary of the publications reviewed and a synthesis of results related to the association of each NUF with LUTS are described below.

\section{Diet and LUTS}

Twenty-eight publications met criteria and provided information related to the association between diet and LUTS (Table 4). Diet was assessed by a food-frequency questionnaire (FFQ) in almost all studies. There were five interventional RCTs ${ }^{9-13}$. Only two specifically addressed adding a particular component to improve LUTS ${ }^{10,13}$. The remaining studies analyzed diet and LUTS in the setting of interventions concerning diabetes prevention ${ }^{9}$, constipation reduction ${ }^{11}$, and weight $\operatorname{loss}^{12,14}$. (See detailed summary of publications in Supplemental Tables 2A-D.)

Diet and BPH-Overall, there appears to be a weak association for diet and surgical BPH therapy. A myriad of food types, food groups, micronutrients and macronutrients were evaluated. Consumption of a high-calorie diet, high in starches and red meat may be weakly associated with BPH risk, while a lower-calorie diet, high in vegetables (specifically allium vegetables, e.g. onion and garlic), high in polyunsaturated fats (including specifically eicosapentanoic and docosahexanoic acids) and low in saturated fat may be associated with decreased risk ${ }^{15-20}$. Studies on micronutrients have implicated carotene to decrease risk while zinc may increase risk $^{21,22}$. 
Diet and Urinary Incontinence (UI)—Many studies that assess diet and UI are indirectly evaluating diet through weight loss. The review found two RCTs in women, the first showing intensive lifestyle therapy (including a low calorie diet) decreased UI in prediabetic women ${ }^{9}$ and the second that modest weight reduction (mean $7.8 \mathrm{~kg}$ ) can decrease stress urinary incontinence (SUI) episodes (but not urge) ${ }^{12}$, and that weight loss (5-10\% of body weight) was sufficient to significantly decrease UI episodes ${ }^{14}$. Another RCT specifically looked at urgency incontinence episodes in nursing home patients and found when combined with toileting assistance, exercise and an increase in caloric intake, UI episodes decreased significantly ${ }^{11}$. Another RCT evaluated a diet rich in soy, hypothesized to increase circulating estrogens via phytoestrogens, showed no improvement versus a control diet on overall LUTS or UI. ${ }^{10}$

Studies looking at the association of dietary components and incontinence found consumption of saturated and monounsaturated fats and carbonated beverages may increase the risk of SUI while intake of breads/starches and vegetables may decrease the risk ${ }^{23,24}$. Interestingly, similar to the association seen in BPH, zinc intake was associated with SUI in women as was vitamin B12. ${ }^{23,} 24$ Consumption of phytoestrogens did not affect SUI.

Diet and General Urinary Symptoms-Validated questionnaire use was a common way to test associations between diet and LUTS. Most studies were cross-sectional, and thus, determining causality becomes more difficult given researchers believe lifetime exposure is more important for health than current diet. Clinically significant LUTS was associated with poor overall diet and dietary variety ${ }^{25}$, increase in total caloric intake (adjusting for weight), ${ }^{26,27}$ and sodium intake in men ${ }^{26}$. Protein intake may decrease the risk in men ${ }^{25}$ but increases the risk of storage symptoms in women ${ }^{27}$. In elderly men, consumption of isoflavone (a phytoestrogen) showed a strong correlation with LUTS ${ }^{28}$. However, a single RCT evaluated the effects of flaxseed extract (high in phytoestrogens) on LUTS and noted a dose-dependent and significant decrease in LUTS in men on flaxseed ${ }^{13}$. In a longitudinal cohort study, higher vitamin $\mathrm{C}$ intake at baseline was associated with less progression of storage LUTS but vitamin C supplementation was associated with worse LUTS at five-year follow-up in women ${ }^{29}$.

Diet and OAB - Few studies have directly assessed OAB and diet. There appears to be a weak association with potato/starch consumption ${ }^{30}$. Evaluation of micronutrients suggests higher consumption of vitamin $\mathrm{D}$, protein, and potassium may be protective of OAB in women ${ }^{31}$. High energy/caloric intake in the setting of high glycemic indices and low physical activity may also be a risk factor in women ${ }^{32}$.

\section{Fluid intake and LUTS}

Table 5 summarizes the 21 publications reviewed with more details provided in Supplemental Tables 3A-D.

Fluid Intake and BPH-It is unclear from the few published articles whether there is an association between fluid intake and BPH. In one RCT, 138 men with BPH were randomized to increase fluid intake by $1.5 \mathrm{~L}$ per day versus placebo ${ }^{33}$. There was no 
difference in AUA-SI total, voiding and QOL scores between the groups at six months. However, AUA-SI storage scores were worse in those who increased fluid intake (effect size $=1.3, \mathrm{p}<0.001$ ). In a non-randomized, uncontrolled study, AUA-SI increased from 7.9 to $8.9(\mathrm{p}=0.028)$ after subjects increased fluid intake by $2 \mathrm{~L}$ per day for eight weeks ${ }^{34}$. However, the magnitude of symptom worsening was small and likely clinically insignificant.

Fluid Intake and Nocturia-There is not a clear association between fluid intake and nocturia. In a large $\mathrm{RCT}^{35}, 307$ women were randomized to receive tolterodine versus tolterodine plus behavioral therapy, which included pelvic floor muscle exercise training, bladder control techniques, and fluid management. No difference in numbers of voids at night was found between treatment groups at 10 weeks. However, this RCT did not specifically examine fluid management, since other behavioral therapies were included and all subjects received general information to avoid excessive fluid intake. Similarly a large cohort study in men found no association between night-time fluids and incident nocturia ${ }^{36}$. In contrast, two uncontrolled case series showed fluid restriction improved nocturia ${ }^{37,38 .}$

Fluid Intake and OAB Symptoms-Six out of seven studies on OAB symptoms reported a positive association between fluid intake and urinary frequency/urgency, including two small cross-over RCTs. One randomized 69 women to caffeine restriction plus daily fluid increase to $3 \mathrm{~L}$, compared to caffeine restriction plus fluid decrease to $750 \mathrm{~mL}$ daily ${ }^{39}$. Another included 24 men and women randomized to increase vs. decrease their daily fluid intake by $25 \%$ compared to their baseline ${ }^{40}$. Both trials found significantly increased frequency and urgency symptoms with fluid increase, and decreased frequency and urgency with fluid reductions.

Fluid Intake and UI-Fourteen articles had mixed results on association between fluid intake and UI: six showed positive, one showed negative and seven no correlations. In a small RCT, increasing fluid intake worsened weekly UI episodes, while decreasing fluid intake improved weekly incontinence. In contrast, the Zimmern $\mathrm{RCT}^{35}$ did not show any correlation. One RCT did not yield any useful results, since most patients were not able to adhere to the fluid protocols ${ }^{41}$. Two cohort studies examined new-onset UI: one found consumption of carbonated drinks was associated with new onset SUI, ${ }^{24}$ while another did not show an association between fluid intake and new $\mathrm{UI}^{42}$.

\section{Caffeine Intake and LUTS}

Twenty articles on caffeine intake and LUTS are summarized in Table 6 (detailed summary found in Supplemental Tables 4A-E). Most were observational studies, but two small RCTs were reviewed. Most assessed caffeine intake as self-reported cups of coffee or milligrams of caffeine per day, estimated using a self-reported FFQ or other non-validated questions. Two studies in women focused on urodynamic test findings related to caffeine. One small study found detrusor pressure during filling increased (but other parameters were unchanged) after caffeine ingestion. ${ }^{43}$ Another found detrusor overactivity in women with UI was associated with higher caffeine intake. ${ }^{44}$ 
Caffeine and BPH-It is unclear if caffeine intake and BPH are associated, and evidence reviewed was limited to coffee, not caffeine, intake. Three studies on caffeine intake in men with BPH had conflicting results. Two older case control studies of men with surgicallytreated $\mathrm{BPH}$ found non-significant associations between coffee intake and $\mathrm{BPH}^{45}$, 46 . A large, population-based, cross-sectional study found increasing coffee consumption positively associated with $\mathrm{BPH}^{47}$.

Caffeine and Nocturia-Coffee consumption was not associated with nocturia in men or women, as tested in a cohort study of men and a large cross-sectional study of women ${ }^{48,49}$. In contrast, the same cross-sectional study found tea intake was associated with increasing nocturia, although the increased risk was small (OR 1.2) and only in women drinking three or more cups of tea daily. Another large cross sectional study found women with nocturia were less likely to drink caffeinated beverages after $6 \mathrm{pm}^{50}$. This finding highlights the limitations of cross-sectional analyses, since women who have nocturia may be likely to avoid caffeinated beverages in the evening.

Caffeine and OAB/LUTS - Small, randomized interventional studies provide limited evidence that caffeine reduction may decrease OAB symptoms in women. In two RCT (mostly women), caffeine reduction was associated with reduced urinary frequency, urgency, and OAB QOL scores ${ }^{51,52}$. Caffeine restriction was not associated with changes in frequency/urgency in a third uncontrolled study ${ }^{39}$. Coffee consumption in a large crosssectional study was not associated with urgency, but women reporting tea intake (three or more cups daily) were more likely to report urgency ${ }^{53}$. Fewer studies have evaluated caffeine and $\mathrm{OAB}$ in men, though one large, cross-sectional study found caffeine intake was not associated with increased LUTS ${ }^{54}$.

Caffeine and UI-Overall evidence may suggest a weak positive association between caffeine and UI, but there are conflicting results for UI types, and studies in men are lacking. Four interventional studies (two randomized, two uncontrolled) found no impact for caffeine reduction on $\mathrm{UI}^{39,51,52,55}$. A longitudinal study in women found caffeine associated with frequent UI and urgency urinary incontinence (UUI), but only in women with the greatest caffeine intake ${ }^{56}$. Caffeine intake was not associated with UI progression when analyzed in the same longitudinal data ${ }^{57}$. Mixed results were found in several large cross-sectional studies in women, with one finding coffee and tea intake associated with SUI, and tea intake with overall UI (but not UUI), while another found no association between tea or coffee consumption and any type of $\mathrm{UI}^{53,58,59}$. One large cross-sectional study of men found the highest level of caffeine intake associated with moderate to severe UI (but not with any $\mathrm{UI})^{60}$.

\section{Alcohol and LUTS}

Twenty-six articles on alcohol intake and LUTS were identified, reviewed and graded (Table 7). Study details are presented in Supplemental Tables 5A-E. Most publications assessed alcohol intake as self-reported drinks per day, week or month, or grams of alcohol consumed based on the subject's self-report. Four articles analyzed alcohol type (beer, wine, spirits) as well as total consumption. 
Alcohol and BPH and LUTS in Men-Results for BPH and BPH surgery were consistent among seven of eight articles reviewed, with a decrease in BPH diagnosis or surgery in men who drank alcohol compared to non-drinkers. The association between alcohol intake and the reduction in BPH diagnosis and surgery was particularly strong for modest alcohol consumption (defined as 1-3 drinks per day), with this consumption level having the greatest $\mathrm{BPH}$ reduction compared to non-drinkers.

LUTS, UI and nocturia in men alone were assessed in 14 articles and had relatively consistent findings with modest drinking associated with fewer symptoms compared to nondrinkers in all but two articles. Heavy alcohol consumption (defined as self-reported alcoholism, $>72 \mathrm{~g} /$ day [ $>5.1$ drinks a day] or $>40 \mathrm{~g} /$ day [ $>2.9$ drinks a day]), however, appears to have a negative effect with an increase in incontinence, obstructive and irritative LUTS ${ }^{61}$. This "J-shaped" association of alcohol intake and LUTS was most clearly seen in a cross-sectional study of 30,196 Korean men participating in a comprehensive health examination, where the odds of moderate or severe AUA-SI scores were lowest among modest drinkers $(0-10 \mathrm{~g} /$ day $)$ compared to non-drinkers and highest in men who drank $>40 \mathrm{~g} / \mathrm{day}^{62}$.

Alcohol and Nocturia-Only two articles assessed nocturia. In a group with both genders, no association was found between alcohol intake and nocturia ${ }^{63}$, whereas in a single article including only men, modest alcohol intake had the lowest risk of moderate or severe nocturia ${ }^{48}$

Alcohol and $\mathrm{OAB} / \mathrm{UI}-\mathrm{Among}$ three articles assessing $\mathrm{OAB}$ there were inconsistent results. Results from the BACH study showed inconsistent findings by intake level and symptom subtype with few groups achieving statistical significance ${ }^{64}$, whereas an interview study of 833 elderly individuals found greater odds of urgency and frequency among current drinkers compared to non-drinkers, but not ex-drinkers ${ }^{65}$. In four articles assessing UI no association was found between any type of UI and alcohol intake.

\section{Tobacco and LUTS}

Forty-four articles on tobacco use and LUTS were systematically reviewed (Table 8, with detailed summary included in Supplemental Tables 6A-E.). Most were cross-sectional, but some cohort and case-controls studies were reviewed. Tobacco use was almost always studied as self-reported current cigarette smoking.

Tobacco and BPH-Eight of 12 articles reported no association between BPH and tobacco. Four found a negative association between heavy or current smoking and $\mathrm{BPH}$, but no trend in the association with quantity of cigarettes smoked ${ }^{47,66-68}$.

Tobacco and Nocturia-Evidence regarding nocturia was inconsistent. Of six studies, one showed a positive association between current smoking and nocturia in women ${ }^{50}$, two showed a negative association in women ${ }^{69}$ and men $^{68}$, one showed a negative association with heavy current smoking (but not lighter current smoking) in a sample of men and women ${ }^{70}$. Two studies showed no association ${ }^{48,71}$. 
Tobacco and OAB - Within the broad category of evidence for OAB or LUTS in general, there are some consistent and some inconsistent findings. A small amount of evidence suggests former and/or current smoking is related to frequency in women. Two studies showed a positive association between urgency and current tobacco use, ${ }^{71,72}$ while two did not ${ }^{69,73}$. A single study each showed no association with irritative symptoms in men ${ }^{61}$ or voiding symptoms in men and women ${ }^{74}$, but a positive association with obstructive symptoms in men ${ }^{61}$ and storage symptoms in women ${ }^{74}$. LUTS in general was the most common outcome in this category but had the most inconsistent results.

Tobacco and UI-We found no evidence to review regarding UI in men. In women, studies reported inconsistent results. Some studies provided evidence of a positive association between tobacco use and $\mathrm{SUI}^{75,76}$, UUI and mixed incontinence ${ }^{77}$, motor incontinence $^{75}$, and incontinence of any (unspecified) type ${ }^{59,77,78}$. Six studies showed no associations $69,71,73,79-81$, and one showed a negative association between occasional UI and current smoking ${ }^{78}$. In addition, Hannested $2003^{59}$ showed mixed results between current, former, and heavy smoking and various measures of incontinence. The two studies that examined severe UI showed a positive association ${ }^{59,78}$.

Tobacco and Other LUTS Measures-A few studies focused on other parameters. Single studies showed positive associations between smoking and women's maximum cough spike ${ }^{82}$, cough leak point pressure and maximal intravesical pressures generated by $\operatorname{cough}^{83}$, and men's estradiol levels ${ }^{84}$. Two studies showed evidence that male smokers were at lower risk of low urinary flow rates ${ }^{85,86}$, but another found no association ${ }^{84}$. No associations were found between tobacco use and women's maximum closure pressure, mean pressure transmission ratio, degree of urethral axis with stress ${ }^{82}$, or men's testosterone, dehydroepiandrosterone (DHEA), or DHEA-S levels or prostate weight. ${ }^{84}$

\section{Discussion}

We systematically reviewed the literature studying associations between LUTS and daily behaviors, including diet, fluid intake, and caffeine, alcohol and tobacco use. We identified 110 articles meeting our eligibility criteria, graded their evidence, and summarized these findings by population and LUTS condition studied. Overall, relatively few, largely observational studies were eligible $(<50$ per factor) and evidence quality was low.

Based on our review, few definitive conclusions about associations could be made. In observational studies of men, modest alcohol use (compared to non-use) was associated with less likelihood of a BPH diagnosis and fewer LUTS. As alcohol intake of this level falls within federal recommendations, this might be considered a reasonable recommendation in clinical practice. Fluid intake was positively associated with urinary frequency and urgency symptoms in men and women in two small interventional studies and in observational studies of mixed quality. These findings support the inclusion of fluid management within the behavioral strategies recommended as first-line treatments for $\mathrm{OAB}^{4}$. In other areas of our review, inconsistent results or lack of evidence precluded conclusions about associations between NUF and LUTS. 
Strengths of this effort include our standardized protocol used for screening citations, determining eligibility for inclusion and abstracting study results. We used an accepted and widely-used system for grading the evidence of the included studies. When possible, we attempted to synthesize results to help clarify clinical usefulness of the literature.

One study limitation is that most of the evidence was from observational studies, and we did not attempt meta-analyses due to the heterogeneous search results. Few studies in any single area included similar enough outcomes and exposures that statistical integration would be useful or valid. Given our results included lower evidence levels, caution must be taken in making clinical recommendations based on these findings ${ }^{89,} 90$. However, systematic review of observational studies may be an important alternative when RCTs cannot produce the evidence needed or would be unethical ${ }^{90}$. For example, a dietary factor may require an extended duration of exposure to cause LUTS, which could not be feasibly assessed in an RCT. In another example, RCTs measuring the impact of tobacco on LUTS would be unethical.

Another weakness to much of the evidence included in this review is the use of self-report measurement to assess exposures. Most of the reviewed articles assessed exposures using self-report, often by interview or questionnaire. Thus, the associations between the NUF and LUTS summarized here are subject to limitations of participants' memory and social desirability effects. Other measurement tools, such as electronic diaries or biomarkers, may provide more valid assessment of exposure in future studies. However, any research in lifestyle factors is likely to face challenges obtaining accurate and unbiased measurements of these factors.

The challenges in performing research on lifestyle factors may partially explain the lack of higher quality evidence available. Despite this (or perhaps because of this), we feel a systematic review of this observational data is important to summarize the evidence available (even if lower quality) and to highlight the lack of evidence in this important topic area. We hope results from this review will spur additional research on lifestyle changes that may modify and/or prevent LUTS. On the other hand, we also acknowledge that strong evidence may never exist for some of these research questions. In these cases, LUTS providers should make a practical assessment of the evidence available and consider the individual situation of each patient in making clinical recommendations.

\section{Conclusions}

Systematic literature review revealed that evidence supporting associations between LUTS and diet, fluid intake, and caffeine, alcohol and tobacco use is sparse. The data available are largely observational and generally lower quality. Given these factors are often modifiable and are frequently included in management recommendations by LUTS care providers, more and higher quality evidence is needed to better understand their impact on LUTS.

\section{Supplementary Material}

Refer to Web version on PubMed Central for supplementary material. 


\section{Acknowledgments}

Source of Funding

This is publication number 3 of the Symptoms of Lower Urinary Tract Dysfunction Research Network (LURN).

This study is supported by the National Institute of Diabetes \& Digestive \& Kidney Diseases through cooperative agreements (grants DK097780, DK097772, DK097779, DK099932, DK100011, DK100017, DK097776, DK099879).

\section{Appendix}

The following individuals were instrumental in the planning and conduct of this study at each of the participating institutions:

Duke University, Durham, North Carolina (DK097780): PI: Cindy Amundsen, MD, Kevin Weinfurt, PhD; Co-Is: Kathryn Flynn, PhD, Matthew O. Fraser, PhD, Todd Harshbarger, $\mathrm{PhD}$, Aaron Lentz, MD, Drew Peterson, MD, Nazema Siddiqui, MD, Alison Weidner, MD; Study Coordinators: Carrie Dombeck, MA, Robin Gilliam, MSW, Akira Hayes, Shantae McLean, MPH

University of Iowa, Iowa City, IA (DK097772): PI: Karl Kreder, MD, MBA, Catherine S Bradley, MD, MSCE, Co-Is: Bradley A. Erickson, MD, MS, Susan K. Lutgendorf, PhD, Vince Magnotta, PhD, Michael A. O'Donnell, MD, Vivian Sung, MD; Study Coordinators: Ahmad Alzubaidi, Andrea Lopez, Linda Moss, RN, BSN, CCRC

Northwestern University, Chicago, IL (DK097779): PI: David Cella, PhD; Co-Is: James Griffith, PhD, Kimberly Kenton, MD, MS, Christina Lewicky-Gaupp, MD, Todd Parrish, PhD, Jennie Yu Fan Chan, MD; Study Coordinators: Sarah Buono, Maria Corona, Beatriz Menendez, Alexis Siurek, Meera Tavathia, Veronica Venezuela

NorthShore University Health System, Evanston, IL (DK097779): PI: Brian T. Helfand, MD, PhD; Study Coordinators: Jasmine Nero, Pooja Talaty

University of Michigan Health System, Ann Arbor, MI (DK099932): PI: J Quentin Clemens, MD, FACS, MSCI; Co-Is: Mitch Berger, MD, PhD, John DeLancey, MD, Dee Fenner, MD, Rick Harris, MD, Steve Harte, PhD, Anne Pelletier-Cameron, MD, John Wei, MD; Study Coordinators: Morgen Barroso, Linda Drnek, Greg Mowatt, Julie Tumbarello

University of Washington, Seattle Washington (DK100011): PI: Claire Yang, MD; Co-I: John L. Gore, MD, MS; Study Coordinators: Alice Liu, MPH, Brenda Vicars, RN

Washington University in St. Louis, St. Louis Missouri (DK100017): PI: Gerald Andriole, MD, Henry Lai; Co-I: Joshua Shimony, MD, PhD; Study Coordinators: Susan Mueller, RN, BSN, Heather Wilson, LPN, Aleksandra Klim, RN, MHS, CCRC

National Institute of Diabetes and Digestive and Kidney Diseases, Division of Kidney, Urology, and Hematology, Bethesda, MD: Project Scientist: Ziya Kirkali MD; Project Officer: John Kusek, PhD; NIH Personnel: Tamara Bavendam, MD, Robert Star, MD, Jenna Norton, MPH 
Arbor Research Collaborative for Health, Data Coordinating Center (DK097776 and DK099879): PI: Robert Merion, MD, FACS; Co-Is: Brenda Gillespie, PhD, Victor Andreev, PhD, DSc; Project Manager: Melissa Fava, MPA, PMP; Clinical Study Process Manager: Peg Hill-Callahan, BS, LSW; Clinical Monitor: Timothy Buck, BS, CCRP; Research Analysts: Margaret Helmuth, MA, Jon Wiseman, MS; Project Associate: Julieanne Lock, MLitt, BA

\section{References}

1. Kupelian V, Wei JT, O'Leary MP, et al. Prevalence of lower urinary tract symptoms and effect on quality of life in a racially and ethnically diverse random sample: the Boston Area Community Health (BACH) Survey. Arch Intern Med. 2006; 166:2381. [PubMed: 17130393]

2. Coyne KS, Sexton CC, Thompson CL, et al. The prevalence of lower urinary tract symptoms (LUTS) in the USA, the UK and Sweden: results from the Epidemiology of LUTS (EpiLUTS) study. BJU Int. 2009; 104:352. [PubMed: 19281467]

3. Coyne KS, Wein AJ, Tubaro A, et al. The burden of lower urinary tract symptoms: evaluating the effect of LUTS on health-related quality of life, anxiety and depression: EpiLUTS. BJU Int. 2009; 103(Suppl 3):4.

4. Gormley EA, Lightner DJ, Burgio KL, et al. Diagnosis and treatment of overactive bladder (nonneurogenic) in adults: AUA/SUFU guideline. J Urol. 2012; 188:2455. [PubMed: 23098785]

5. Yang CC, Weinfurt KP, Merion RM, et al. Symptoms of Lower Urinary Tract Dysfunction Research Network. J Urol. 2016; 196:146. [PubMed: 26784646]

6. Moher D, Liberati A, Tetzlaff J, et al. Preferred reporting items for systematic reviews and metaanalyses: the PRISMA statement. PLoS Med. 2009; 6:e1000097. [PubMed: 19621072]

7. Medicine, C. f. E.-B. Oxford Centre for Evidence-based Medicine - Levels of Evidence (March 2009). 2009

8. Abrams P, Khoury S. International Consultation on Urological Diseases: Evidence-based medicine overview of the main steps for developing and grading guideline recommendations. Neurourol Urodyn. 2010; 29:116. [PubMed: 20025028]

9. Brown JS, Wing R, Barrett-Connor E, et al. Lifestyle intervention is associated with lower prevalence of urinary incontinence: the Diabetes Prevention Program. Diabetes Care. 2006; 29:385. [PubMed: 16443892]

10. Manonai J, Songchitsomboon S, Chanda K, et al. The effect of a soy-rich diet on urogenital atrophy: a randomized, cross-over trial. Maturitas. 2006; 54:135. [PubMed: 16297576]

11. Schnelle JF, Leung FW, Rao SS, et al. A controlled trial of an intervention to improve urinary and fecal incontinence and constipation. J Am Geriatr Soc. 2010; 58:1504. [PubMed: 20653804]

12. Subak LL, Wing R, West DS, et al. Weight loss to treat urinary incontinence in overweight and obese women. N Engl J Med. 2009; 360:481. [PubMed: 19179316]

13. Zhang W, Wang X, Liu Y, et al. Effects of dietary flaxseed lignan extract on symptoms of benign prostatic hyperplasia. J Med Food. 2008; 11:207. [PubMed: 18358071]

14. Wing RR, Creasman JM, West DS, et al. Improving urinary incontinence in overweight and obese women through modest weight loss. Obstet Gynecol. 2010; 116:284. [PubMed: 20664387]

15. Araki H, Watanabe H, Mishina T, et al. High-risk group for benign prostatic hypertrophy. Prostate. 1983; 4:253. [PubMed: 6189108]

16. Bravi F, Bosetti C, Dal Maso L, et al. Food groups and risk of benign prostatic hyperplasia. Urology. 2006; 67:73. [PubMed: 16413336]

17. Chyou PH, Nomura AM, Stemmermann GN, et al. A prospective study of alcohol, diet, and other lifestyle factors in relation to obstructive uropathy. Prostate. 1993; 22:253. [PubMed: 7683816]

18. Galeone C, Pelucchi C, Talamini R, et al. Onion and garlic intake and the odds of benign prostatic hyperplasia. Urology. 2007; 70:672. [PubMed: 17991535] 
19. Kristal AR, Arnold KB, Schenk JM, et al. Dietary patterns, supplement use, and the risk of symptomatic benign prostatic hyperplasia: results from the prostate cancer prevention trial. Am J Epidemiol. 2008; 167:925. [PubMed: 18263602]

20. Suzuki S, Platz EA, Kawachi I, et al. Intakes of energy and macronutrients and the risk of benign prostatic hyperplasia. Am J Clin Nutr. 2002; 75:689. [PubMed: 11916755]

21. Lagiou P, Wuu J, Trichopoulou A, et al. Diet and benign prostatic hyperplasia: a study in Greece. Urology. 1999; 54:284. [PubMed: 10443726]

22. Tavani A, Longoni E, Bosetti C, et al. Intake of selected micronutrients and the risk of surgically treated benign prostatic hyperplasia: a case-control study from Italy. Eur Urol. 2006; 50:549. [PubMed: 16442205]

23. Dallosso H, Matthews R, McGrother C, et al. Diet as a risk factor for the development of stress urinary incontinence: a longitudinal study in women. Eur J Clin Nutr. 2004; 58:920. [PubMed: 15164113]

24. Dallosso HM, McGrother CW, Matthews RJ, et al. The association of diet and other lifestyle factors with overactive bladder and stress incontinence: a longitudinal study in women. BJU Int. 2003; 92:69. [PubMed: 12823386]

25. Erickson BA, Vaughan-Sarrazin M, Liu X, et al. Lower urinary tract symptoms and diet quality: findings from the 2000-2001 National Health and Nutrition Examination Survey. Urology. 2012; 79:1262. [PubMed: 22656406]

26. Maserejian NN, Giovannucci EL, McKinlay JB. Dietary macronutrients, cholesterol, and sodium and lower urinary tract symptoms in men. Eur Urol. 2009; 55:1179. [PubMed: 18692303]

27. Maserejian NN, McVary KT, Giovannucci EL, et al. Dietary macronutrient intake and lower urinary tract symptoms in women. Ann Epidemiol. 2011; 21:421. [PubMed: 21421330]

28. Wong SY, Lau WW, Leung PC, et al. The association between isoflavone and lower urinary tract symptoms in elderly men. Br J Nutr. 2007; 98:1237. [PubMed: 17640419]

29. Curto TM, Giovannucci EL, McKinlay JB, et al. Associations between supplemental or dietary intake of vitamin C and severity of lower urinary tract symptoms. BJU Int. 2015; 115:134. [PubMed: 24472044]

30. Dallosso HM, Matthews RJ, McGrother CW, et al. The association of diet and other lifestyle factors with the onset of overactive bladder: a longitudinal study in men. Public Health Nutr. 2004; 7:885. [PubMed: 15482614]

31. Dallosso HM, McGrother CW, Matthews RJ, et al. Nutrient composition of the diet and the development of overactive bladder: a longitudinal study in women. Neurourol Urodyn. 2004; 23:204. [PubMed: 15098215]

32. McGrother CW, Donaldson MM, Thompson J, et al. Etiology of overactive bladder: a diet and lifestyle model for diabetes and obesity in older women. Neurourol Urodyn. 2012; 31:487. [PubMed: 22374635]

33. Spigt M, van Schayck O, Knipschild P, et al. Is it possible to improve elderly male bladder function by having them drink more water? A randomized trial of effects of increased fluid intake/urine output on male lower urinary tract function. Urology. 2006; 68:1031. [PubMed: 17113895]

34. Spigt MG, Knottnerus JA, van de Beek C, et al. Short-term effects of increased urine output on male bladder function and lower urinary tract symptoms. Urology. 2004; 64:499. [PubMed: 15351579]

35. Zimmern P, Litman HJ, Mueller E, et al. Effect of fluid management on fluid intake and urge incontinence in a trial for overactive bladder in women. BJU Int. 2010; 105:1680. [PubMed: 19912207]

36. Johnson TM 2nd, Sattin RW, Parmelee P, et al. Evaluating potentially modifiable risk factors for prevalent and incident nocturia in older adults. J Am Geriatr Soc. 2005; 53:1011. [PubMed: 15935026]

37. Soda T, Masui K, Okuno H, et al. Efficacy of nondrug lifestyle measures for the treatment of nocturia. J Urol. 2010; 184:1000. [PubMed: 20643422]

38. Tani M, Hirayama A, Torimoto K, et al. Guidance on water intake effectively improves urinary frequency in patients with nocturia. Int J Urol. 2014; 21:595. [PubMed: 24405404] 
39. Swithinbank L, Hashim H, Abrams P. The effect of fluid intake on urinary symptoms in women. J Urol. 2005; 174:187. [PubMed: 15947624]

40. Hashim H, Abrams P. How should patients with an overactive bladder manipulate their fluid intake? BJU Int. 2008; 102:62. [PubMed: 18284414]

41. Dowd TT, Campbell JM, Jones JA. Fluid intake and urinary incontinence in older communitydwelling women. J Community Health Nurs. 1996; 13:179. [PubMed: 8916607]

42. Townsend MK, Jura YH, Curhan GC, et al. Fluid intake and risk of stress, urgency, and mixed urinary incontinence. Am J Obstet Gynecol. 2011; 205:73 e1. [PubMed: 21481835]

43. Creighton SM, Stanton SL. Caffeine: does it affect your bladder? Br J Urol. 1990; 66:613. [PubMed: 2265333]

44. Arya LA, Myers DL, Jackson ND. Dietary caffeine intake and the risk for detrusor instability: a case-control study. Obstet Gynecol. 2000; 96:85. [PubMed: 10862848]

45. Morrison AS. Prostatic hypertrophy in greater Boston. J Chronic Dis. 1978; 31:357. [PubMed: 81213]

46. Morrison AS. Risk factors for surgery for prostatic hypertrophy. Am J Epidemiol. 1992; 135:974. [PubMed: 1375808]

47. Gass R. Benign prostatic hyperplasia: the opposite effects of alcohol and coffee intake. BJU Int. 2002; 90:649. [PubMed: 12410741]

48. Shiri R, Hakama M, Hakkinen J, et al. The effects of lifestyle factors on the incidence of nocturia. J Urol. 2008; 180:2059. [PubMed: 18804241]

49. Tettamanti G, Nyman-Iliadou A, Pedersen NL, et al. Influence of smoking, coffee, and tea consumption on bladder pain syndrome in female twins. Urology. 2011; 77:1313. [PubMed: 21439616]

50. Asplund R, Aberg HE. Nocturia in relation to body mass index, smoking and some other lifestyle factors in women. Climacteric. 2004; 7:267. [PubMed: 15669551]

\section{Abbreviation Key}

$\begin{array}{ll}\text { AUA-SI } & \text { American Urological Association Symptom Index } \\ \text { BPH } & \text { benign prostatic hyperplasia } \\ \text { DHEA } & \text { dehydroepiandrosterone } \\ \text { FFQ } & \text { food-frequency questionnaire } \\ \text { LURN } & \text { Symptoms of Lower Urinary Tract Dysfunction Research Network } \\ \text { LUTS } & \text { lower urinary tract symptoms } \\ \text { NIDDK } & \text { National Institute of Diabetes and Digestive and Kidney Diseases } \\ \text { NUF } & \text { non-urologic factors } \\ \text { OAB } & \text { overactive bladder } \\ \text { PRISMA } & \text { Preferred Reporting Items for Systematic Reviews and Meta-Analyses } \\ \text { QOL } & \text { quality of life } \\ \text { RCT } & \text { randomized clinical trial } \\ \text { SUI } & \text { stress urinary incontinence } \\ \text { UI } & \text { urinary incontinence }\end{array}$


UUI urgency urinary incontinence 


\section{Key Point Summary}

- Expert consensus of the reviewed literature suggests a balanced low-calorie/ low-saturated fat diet in a physically active, non-obese person will decrease the lifetime likelihood of developing LUTS and/or BPH, but current evidence for associations between diet and individual dietary constituents and LUTS is mixed and suggests associations, if present, are weak.

- $\quad$ Fluid intake is associated with urinary frequency and urgency in men and women and the association is bi-directional. Evidence supports the use of fluid reduction to manage urinary urgency (as in the AUA Guideline on $\mathrm{OAB})^{4}$. Given potential risks from dehydration, recommendations that patients reduce their fluid intake by $25 \%$, providing they do not drink $<1 \mathrm{~L} /$ day, seem reasonable ${ }^{87}$. Relationships between UI, nocturia and fluid intake are less conclusive.

- We found inconsistent associations between caffeine intake and BPH and nocturia. Mixed evidence suggests caffeine reduction may reduce urinary frequency and urgency in women (small effects). Conflicting results related to caffeine and overall UI and UI types suggests any association if present is weak. The small number of studies focused on caffeine and LUTS in men (particularly OAB and UI symptoms) made it difficult to interpret these results.

- Moderate/modest alcohol consumption in men is associated with a reduced risk of BPH and BPH surgery, as well as decreased LUTS compared to nondrinkers, however excessive alcohol intake above the recommended threshold of healthy consumption is associated with worse LUTS in men. These results are consistent with the U.S. Department of Health and Human Services' recommendation on alcohol consumption for men (two drinks or less/day) $)^{88}$. In contrast, we found no evidence for women that avoidance of alcohol reduces the risk of UI or LUTS.

- We did not find strong evidence that smoking increases UI. Indeed, we found mixed, and therefore weak, evidence for any associations between tobacco use and LUTS. There was perhaps a positive association between smoking and urinary frequency in women, based on limited evidence. 


\section{Table 1}

Criteria for excluding article from the systematic review

\begin{tabular}{|l|c|}
\hline Reason for Exclusion & Number \\
\hline No relevant non-urologic factor studied & 158 \\
\hline No relevant LUTS or LUT condition (including prostate cancer) & 83 \\
\hline LUTS studied as treatment result or adverse effect (e.g. post-prostatectomy incontinence) & 24 \\
\hline Sample size <25 (unless RCT design) & 3 \\
\hline Editorial, commentary, non-systematic review & 76 \\
\hline Non-relevant research type (e.g. qualitative studies, instrument development) & 6 \\
\hline Not human subjects research & 9 \\
\hline Pediatric population & 5 \\
\hline Pregnant population & 1 \\
\hline
\end{tabular}

More than one reason for exclusion may be listed for an individual article 
Table 2

Levels of evidence used in grading the articles (modified from the 2009 Oxford Centre for Evidence-based Medicine Levels of Evidence and the International Consultation on Urological Diseases) ${ }^{7,8}$

\begin{tabular}{|c|l|}
\hline Level & Study Design or Type \\
\hline 1a & Systematic review of RCTs \\
\hline b $\mathrm{b}$ & Individual RCT (good quality) \\
\hline $2 \mathrm{a}$ & Systematic review of cohort studies \\
\hline $2 \mathrm{~b}$ & Individual cohort study, low quality RCT \\
\hline $3 \mathrm{a}$ & Systematic review of case control studies \\
\hline $3 \mathrm{~b}$ & Individual case control study \\
\hline $4 \mathrm{a}$ & Good quality case-series, poor quality cohort or case control study \\
\hline $4 \mathrm{~b}$ & Cross-sectional study \\
\hline 5 & Expert opinion \\
\hline
\end{tabular}

RCT Randomized controlled trial 


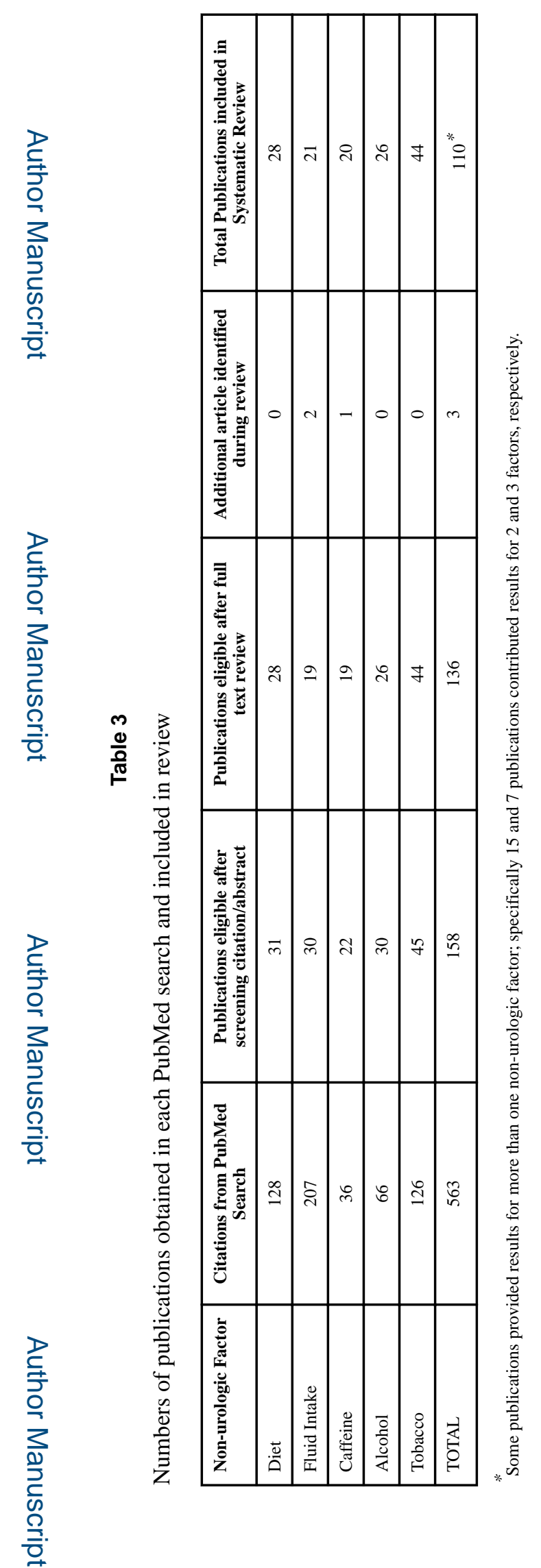

J Urol. Author manuscript; available in PMC 2018 November 01. 


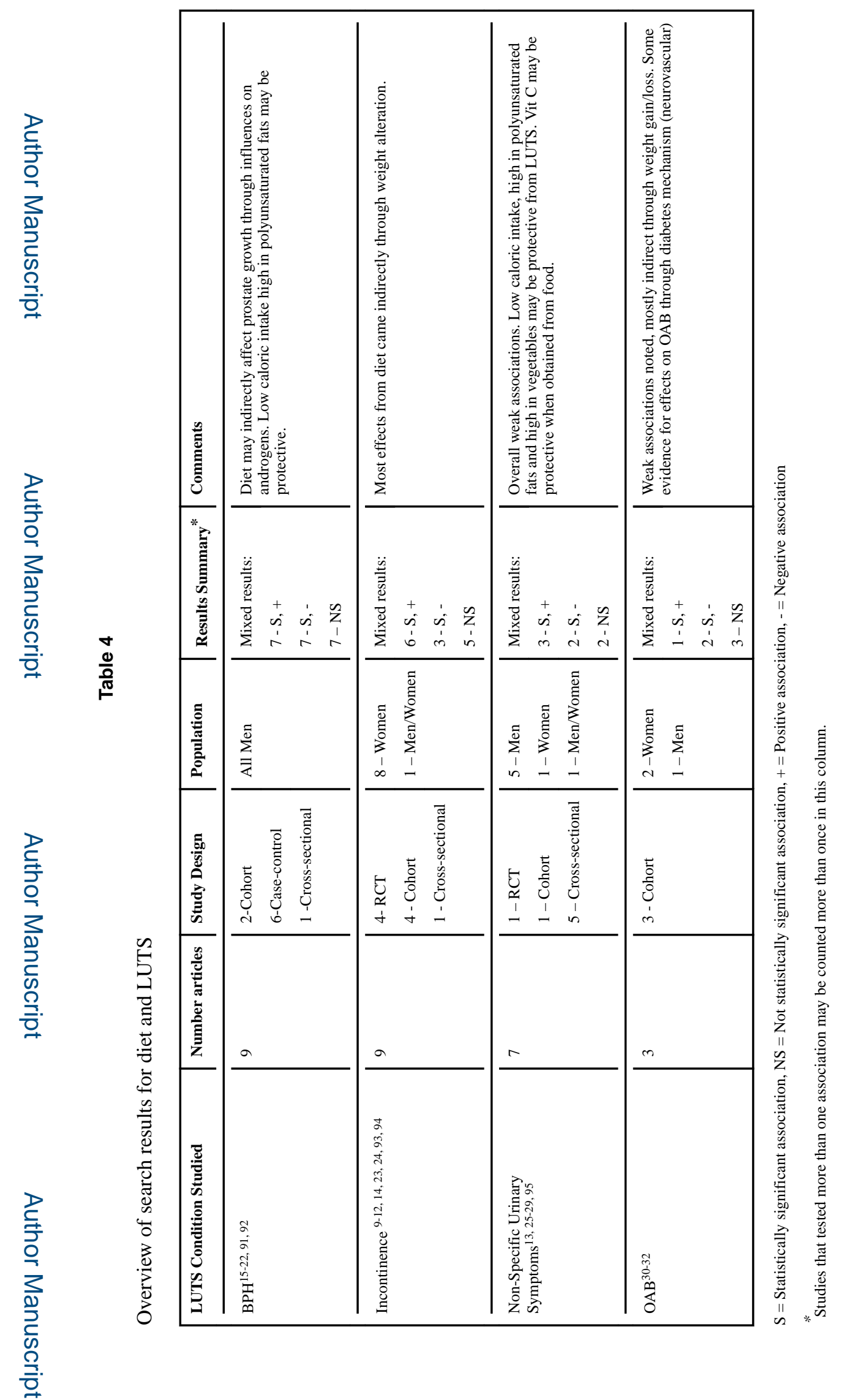

J Urol. Author manuscript; available in PMC 2018 November 01. 


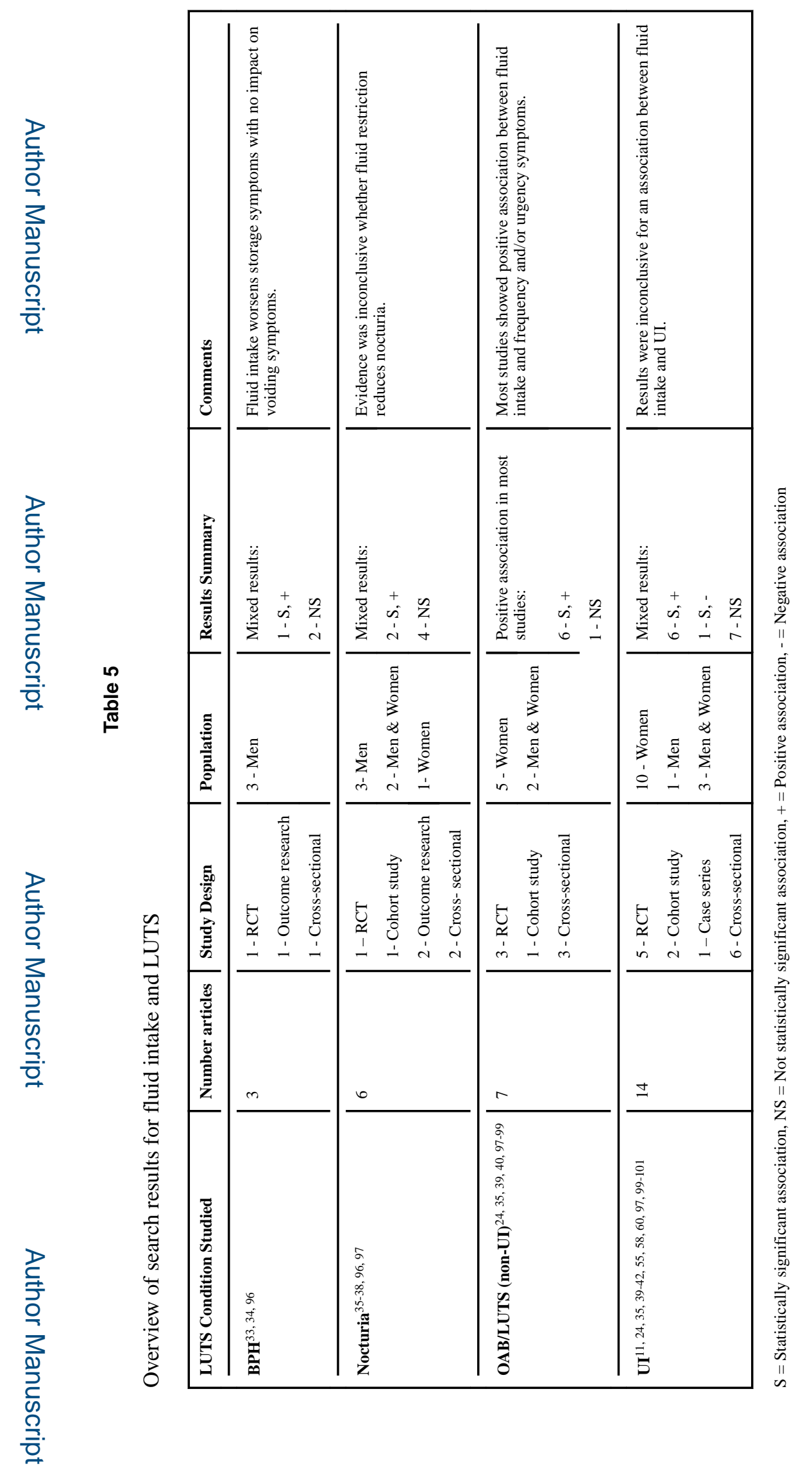

JUrol. Author manuscript; available in PMC 2018 November 01. 


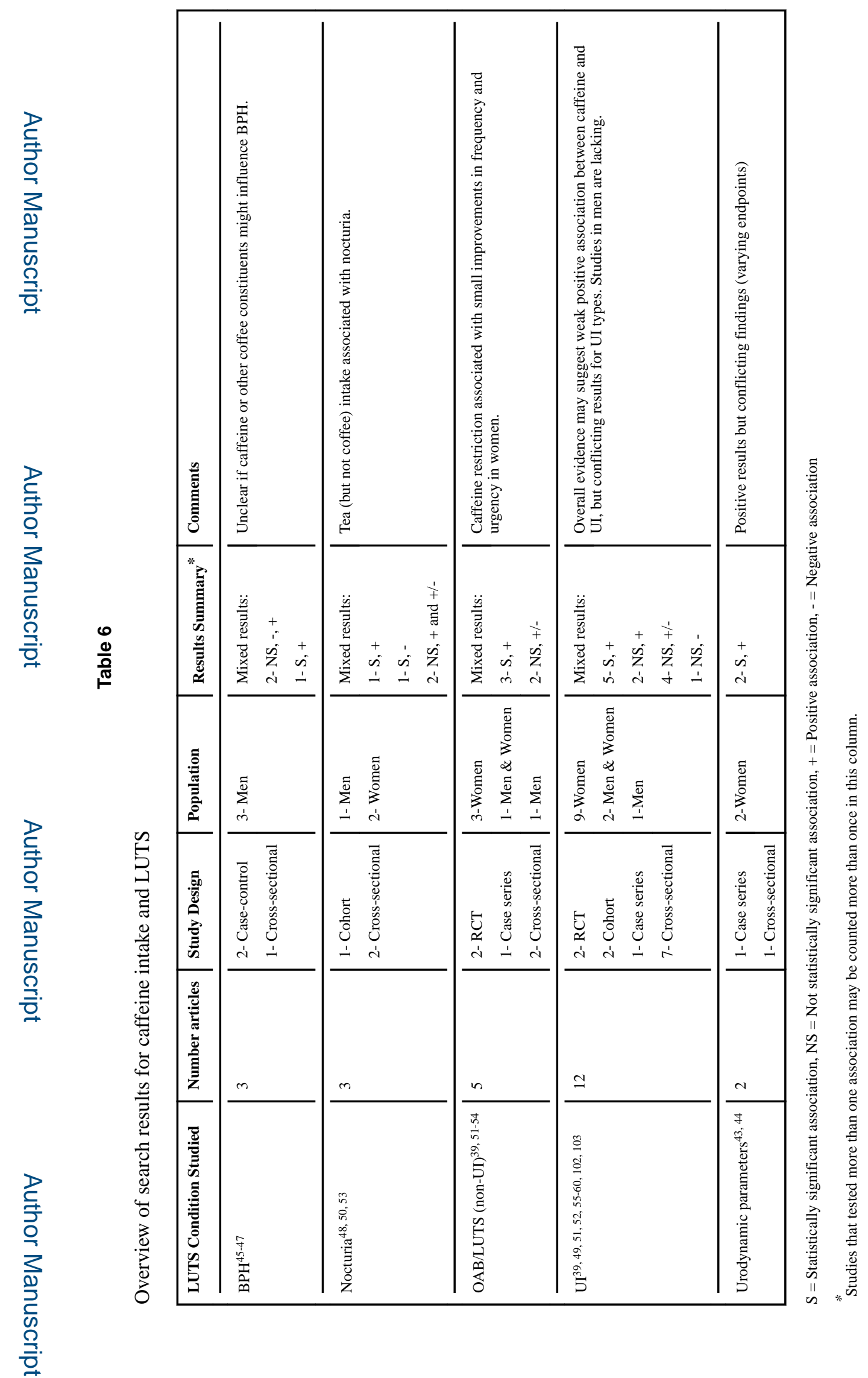

J Urol. Author manuscript; available in PMC 2018 November 01. 


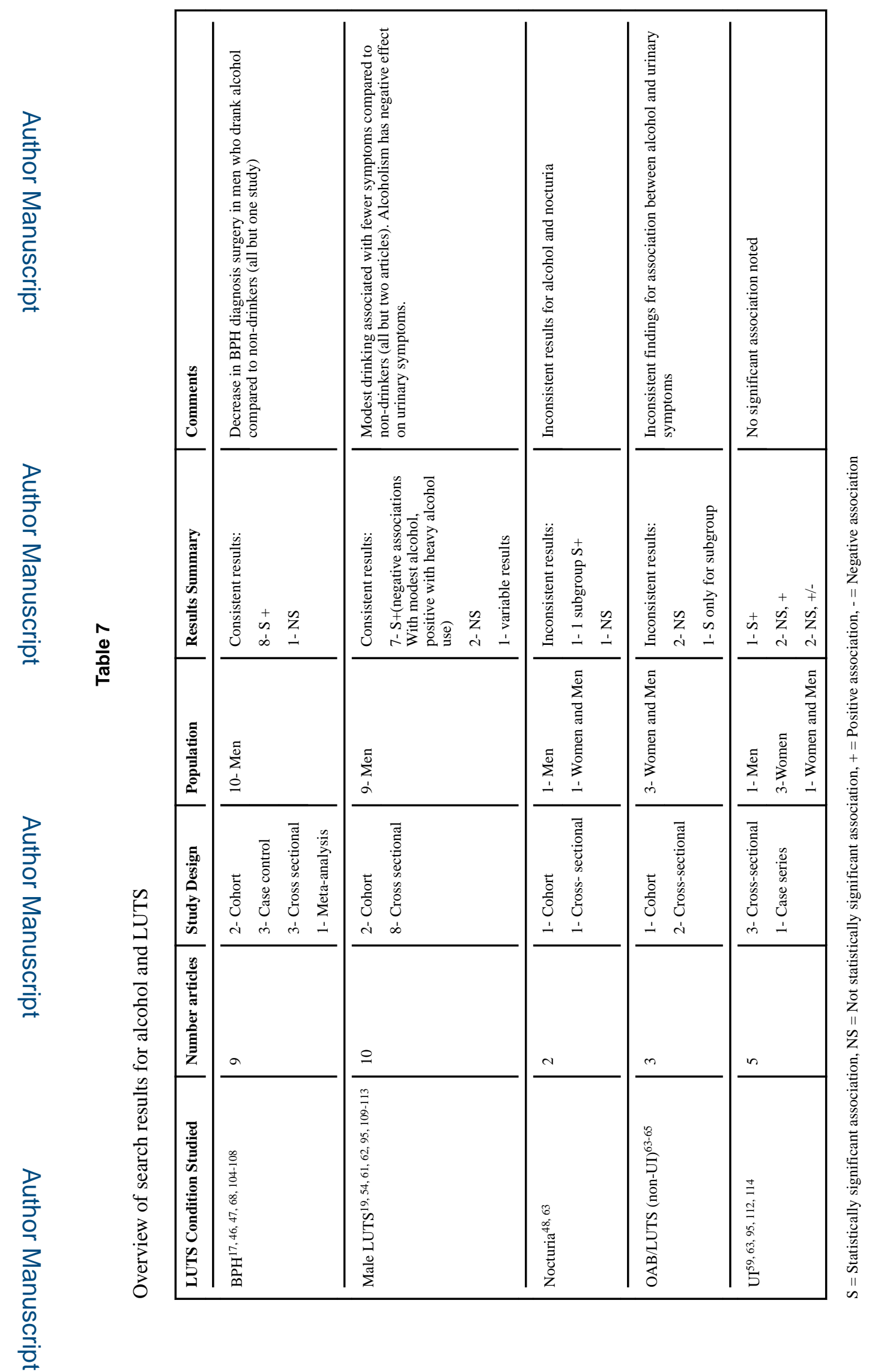

J Urol. Author manuscript; available in PMC 2018 November 01. 


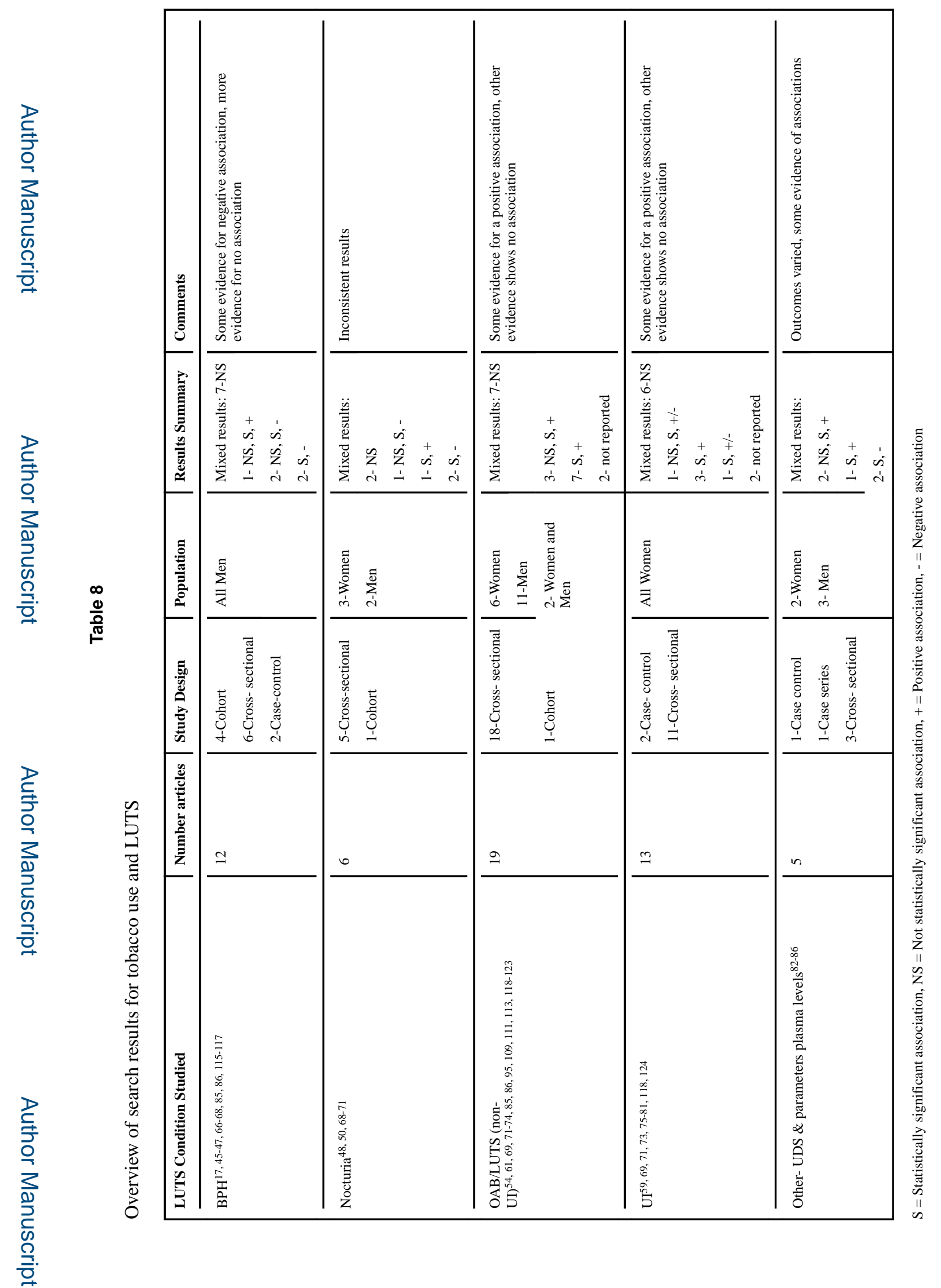

J Urol. Author manuscript; available in PMC 2018 November 01. 